Heinrich Oberreuter - Uwe Kranenpohl Martin Sebaldt (Hrsg.)

Der Deutsche Bundestag im Wandel 
Heinrich Oberreuter - Uwe Kranenpohl Martin Sebaldt (Hrsg.)

\title{
Der Deutsche Bundestag im Wandel
}

\author{
Ergebnisse newerer \\ Parlamentarismusforschung
}

Springer Fachmedien Wiesbaden GmbH 
Die Deutsche Bibliothek - CIP-Einheitsaufnahme

Diese Arbeit wurde unterstützt durch den Deutschen Bundestag.

1. Auflage Juni 2001

Alle Rechte vorbehalten

(C) Springer Fachmedien Wiesbaden 2001

Ursprünglich erschienen bei Leske + Budrich, Opladen 2001

www.westdeutschervlg.de

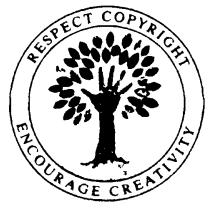

Das Werk einschließlich aller seiner Teile ist urheberrechtlich geschützt. Jede Verwertung außerhalb der engen Grenzen des Urheberrechtsgesetzes ist ohne Zustimmung des Verlags unzulässig und strafbar. Das gilt insbesondere für Vervielfältigungen, Übersetzungen, Mikroverfilmungen und die Einspeicherung und Verarbeitung in elektronischen Systemen.

Die Wiedergabe von Gebrauchsnamen, Handelsnamen, Warenbezeichnungen usw. in diesem Werk berechtigt auch ohne besondere Kennzeichnung nicht zu der Annahme, dass solche Namen im Sinne der Warenzeichen- und Markenschutz-Gesetzgebung als frei zu betrachten wären und daher von jedermann benutzt werden dürften.

Gedruckt auf säurefreiem und chlorfrei gebleichtem Papier.

Umschlaggestaltung: Horst Dieter Bürkle, Darmstadt 


\section{Einführung}

Heinrich Oberreuter, Uwe Kranenpohl, Martin Sebaldt:

Der Deutsche Bundestag: Konstanz und Wandel eines Parlaments.

Zur Einführung

\section{Politische Willensbildung im Deutschen Bundestag: \\ Strukturmuster arbeitsparlamentarischer Praxis}

Manfred Schwarzmeier:

"Nur" Stilfragen? Informale Verhaltensregeln und Handlungsnormen

im Deutschen Bundestag.

Martin Sebaldt:

Das Ringen um die thematische Lufthoheit: Zur Konkurrenz von

Regierungsmehrheit und Opposition in der Gesetzgebungsarbeit

des Deutschen Bundestages

Ekkehard Münzing und Volker Pilz:

Aufgaben, Organisation und Arbeitsweise des Auswärtigen Ausschusses

des Deutschen Bundestages - unter besonderer Berücksichtigung der

12. und 13. Wahlperiode.

Sabine Lemke-Müller:

Interfraktionelle Kooperationen von Parlamentarierinnen im Deutschen Bundestag (Kurzbeitrag)

Politische Entscheidungsprozesse im Gefüge des Bundesstaates:

Der Deutsche Bundestag als Faktor und Zielpunkt politischer Konkurrenz

Roland Lhotta:

Konsens und Konkurrenz in der konstitutionellen Ökonomie bikameraler

Verhandlungsdemokratie: Der Vermittlungsausschuss als effiziente

Institution politischer Deliberation

Sabine Kropp:

Haushaltsentscheidungsprozesse in unterschiedlichen Koalitionsformaten 
Klaus Stüwe:

Das Bundesverfassungsgericht als Vetospieler: Der Erfolg oppositioneller Verfahrensinitiativen vor dem Bundesverfassungsgericht (1951-2000).

\section{Bundestag und Öffentlichkeit:}

Muster und Probleme medialer Präsenz in der Fernsehdemokratie

Stefan Marschall:

Parlamentarische Öffentlichkeit - eine Feldskizze.

Uwe Kranenpohl:

"Die ewige Fahrt zwischen Scylla und Charybdis": Die Öffentlichkeitsarbcit der Fraktionen zwischen parlamentarischer und medialer Funktionslogik

Gregor Mayntz:

Der unbekannte Star: Die Präsenz des Bundestages in den Medien 200

Dietmar Schiller:

Nachrichtenfaktor Parlament: Deutscher Bundestag und britisches House of

Commons im Fernsehen

\section{Bundestag und Gesellschaft:}

Konstanz und Wandel parlamentarischer Repräsentation

Andreas Dörner:

Parlament, politische Kultur und symbolische Form: Zur Semantik des

Deutschen Bundestages im Berliner Reichstag

Frank Brettschneider:

Abgehoben oder bürgernah? Die Responsivität des Deutschen Bundestages .... 258

Martin Sebaldt:

Parlamentarische Demokratie und gesellschaftliche Modernisierung:

Der Deutsche Bundestag im Gefüge organisierter Interessen

seit Mitte der siebziger Jahre 